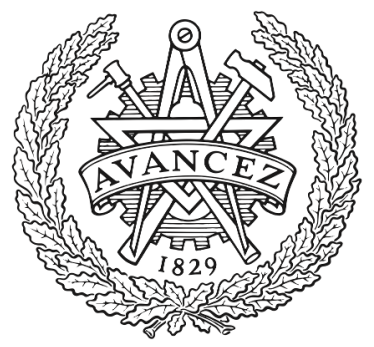

CHALMERS

UNIVERSITY OF TECHNOLOGY

\title{
Nanomaterial interactions with biomembranes: Bridging the gap between soft matter models and biological context
}

Downloaded from: https://research.chalmers.se, 2023-04-26 01:53 UTC

Citation for the original published paper (version of record):

Werner, M., Auth, T., Beales, P. et al (2018). Nanomaterial interactions with biomembranes:

Bridging the gap between soft matter models and

biological context. Biointerphases, 13(2). http://dx.doi.org/10.1116/1.5022145

N.B. When citing this work, cite the original published paper. 


\title{
Nanomaterial interactions with biomembranes: Bridging the gap between soft matter models and biological context
}

\author{
Marco Werner \\ Departament d'Enginyeria Química, Universitat Rovira i Virgili, 26 Avinguda dels Països Catalans,
} 43007 Tarragona, Spain

Thorsten Auth

Theoretical Soft Matter and Biophysics, Institute of Complex Systems and Institute for Advanced Simulation, Forschungszentrum Jülich, 52425 Jülich, Germany

Paul A. Beales

School of Chemistry and Astbury Centre for Structural Molecular Biology, University of Leeds, Leeds

LS2 9JT, United Kingdom

Jean Baptiste Fleury

Experimental Physics, Universität des Saarlandes, 66123 Saarbrücken, Germany

Fredrik Höök

Department of Physics, Chalmers University of Technology, SE-41296 Gothenburg, Sweden

Holger Kress

Department of Physics, University of Bayreuth, 95447 Bayreuth, Germany

Reid C. Van Lehn

Department of Chemical and Biological Engineering, University of Wisconsin-Madison, Madison, Wisconsin 53706

Marcus Müller

Institut für Theoretische Physik, Georg-August Universität, 37077 Göttingen, Germany

\section{Eugene P. Petrov}

Max Planck Institute of Biochemistry, Department of Cellular and Molecular Biophysics, 82152 Martinsried, Germany and Fakultät für Physik, Ludwig-Maximilians-Universität München, Geschwister-Scholl-Platz 1, 80539 München, Germany

\section{Lev Sarkisov}

Institute for Materials and Processes, School of Engineering, The University of Edinburgh, Edinburgh EH9 3FB, United Kingdom

Jens-Uwe Sommer

Leibniz-Institut für Polymerforschung Dresden e.V., Hohe Straße 6, 01069 Dresden, Germany

Vladimir A. Baulin ${ }^{\text {a) }}$

Departament d'Enginyeria Química, Universitat Rovira i Virgili, 26 Avinguda dels Països Catalans, 43007 Tarragona, Spain

(Received 11 January 2018; accepted 19 March 2018; published 3 April 2018)

Synthetic polymers, nanoparticles, and carbon-based materials have great potential in applications including drug delivery, gene transfection, in vitro and in vivo imaging, and the alteration of biological function. Nature and humans use different design strategies to create nanomaterials: biological objects have emerged from billions of years of evolution and from adaptation to their environment resulting in high levels of structural complexity; in contrast, synthetic nanomaterials result from minimalistic but controlled design options limited by the authors' current understanding of the biological world. This conceptual mismatch makes it challenging to create synthetic nanomaterials that possess desired functions in biological media. In many biologically relevant applications, nanomaterials must enter the cell interior to perform their functions. An essential transport barrier is the cell-protecting plasma membrane and hence the understanding of its interaction with nanomaterials is a fundamental task in biotechnology. The authors present open questions in the field of nanomaterial interactions with biological membranes, including: how physical mechanisms and molecular forces acting at the nanoscale restrict or inspire design options; which levels of complexity to include next in computational and experimental models to describe how nanomaterials cross barriers via passive or active processes; and how the biological media and protein corona interfere

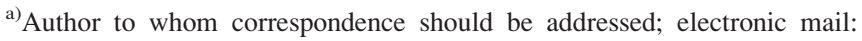

vladimir.baulin@urv.cat
} 
with nanomaterial functionality. In this Perspective, the authors address these questions with the aim of offering guidelines for the development of next-generation nanomaterials that function in biological media. Published by the AVS. https://doi.org/10.1116/1.5022145

\section{INTRODUCTION}

Functional nanomaterials are used in many products of our daily life, from sunscreens to food ${ }^{1}$ but are associated with uncontrolled risks such as nanotoxicity and environmental pollution. ${ }^{2}$ The proper design of "smart" or "intelligent" nanomaterials that perform a desired function in living organisms is an appealing, but challenging task: the complexity of living organisms results from their adaptation to the environment during billions of years of evolution, whereas fabrication of synthetic nanomaterials is usually based on the optimization of a relatively small number of parameters. By offering precise control of design parameters, robustness, and simplicity of construction, synthetic nanomaterials can promise new functions that do not yet exist in the biological world. However, the changes that they induce in complex biological media and their lack of adaptability may compromise the design goals due to degradation or limited biocompatibility. The design of biologically active nanomaterials therefore requires a clear definition of the design goals, the conception and implementation of the material, and methods for testing the materials efficacy. While essential parameters-size, shape, elasticity, composition, and surface properties - of nanomaterials have been identified $^{3-6}$ and their chemical properties can be precisely controlled, the major challenges in nanomaterial design arise in monitoring, understanding, and controlling their interaction with biological media, ranging from specific biological barriers to the immune system.

Using the prototypical example of a transport of nanoobjects into eukaryotic cells, we map out the difficulties of nanomaterial design, and elaborate our opinion on how design obstacles are linked to fundamental questions in understanding the transport into living cells. We also highlight starting points for extending experimental and theoretical models for the prediction of a nanomaterial functionality in biological environments: what are the next degrees of increased complexity that are most important to consider? In Sec. II, we describe where we see the major obstacles for an optimal design flow that integrates all necessary design steps. In Sec. III, we focus on challenges in understanding and exploiting already known mechanisms of nanomaterial transport across plasma membranes, and in Sec. IV, we provide examples that highlight how the presence of biological media challenges theoretical and experimental approaches but also inspires new design concepts.

\section{MISSING LINKS TO BIOLOGICAL CONTEXT}

Modern chemistry allows to synthetize a large variety of nanomaterials with a broad range of architectures (e.g., quantum dots, polymers, nanostars, nanorods, nanodisks, and nanocages), chemical compositions (organic/inorganic, liquid/solid), and surface properties (e.g., decoration with ligands and charges). ${ }^{7}$ A good illustration on recent progress in advanced synthesis is the possibility of dynamically controlling the number of ligands on 23-gold-atom nanoparticle within the so-called molecular surgery. ${ }^{8}$ With fairly high precision, one can control the chemical composition of nanomaterials, the length and order of synthetic peptide sequences, and the architecture, chemistry, and length of synthetic polymers. Yet it is often not clear how chemical properties translate into physical control parameters when embedded in highly complex biological media. When coming from a physical and chemical background, one often focuses on microscopic mechanisms of nanomaterial interactions with model environments, such as single component lipid membranes, although the biological context is essential for formulating critical design goals and testing their functionality. In addition to the extensively discussed protein corona around nanoparticles, ${ }^{9}$ more emphasis should be put on the question of how the protein-crowded environment, cosolvent properties, ionic strength and ion complexation, or $\mathrm{pH}$ modify the conformation and function of soft objects such as polymers or nanogels. In turn, the impact of nanomaterials on the biological environment can be subtle. For example, one has observed that the band structure of metal oxide-based nanomaterials is an important factor for their toxicity. Depending on the band gap, these materials may interfere with the level of oxidative stress and can thereby be toxic. $^{10,11}$

While theoretical and simulation approaches often investigate populations of identical nano-objects with idealized properties such as perfectly smooth spherical nanoparticles or monodisperse polymers, real nano-objects are not so pristine and, for instance, exhibit variations in surface roughness, polydispersity, and heterogeneity within a sample. Since small differences between nano-objects can be critical for their interaction with biological media, different fates are expected already from small variations in their properties, including decomposition into subpopulations due to the complex nature of the interactions.

Due to the Abbé diffraction limit, it is challenging to gain a glimpse at the molecular scale in aqueous solution. However, optical imaging can reveal significant insights into the impact of nano-objects on membrane properties, such as membrane morphology, ${ }^{12}$ dynamics, ${ }^{13}$ and permeability. ${ }^{14}$ Furthermore, structural insights can be gained from spectroscopic methods. ${ }^{15}$ Toward the imaging of individual nanoparticles, alternative approaches, such as stimulated emission depletion microscopy ${ }^{16,17}$ and electron microscopy, ${ }^{18}$ are applied. Microfluidic and electrochemistry methods can also be applied together to monitor individual translocation events of single and clustered nanoparticles across model lipid membranes. ${ }^{19}$ 


\section{CHALLENGES FOR TRANSPORT ACROSS LIPID-BILAYER MEMBRANES}

Whereas there is evidence for insertion and translocation of nano-objects such as cell-penetrating peptides, polymers, or coated nanoparticles across biological and lipid membranes from experiment and simulation, the thermodynamic driving forces and the molecular mechanisms for translocation remain hotly debated. ${ }^{20-23}$ In analogy to other topologyaltering (Fig. 1) membrane processes, such as fusion, fission, and pore formation, ${ }^{24}$ the passage across a membrane can be roughly subdivided into an initial recognition or docking stage and the subsequent penetration, as well as the separation of the object from the membrane. Generally, one may distinguish between active, assisted and passive transport across a membrane. Furthermore, one should distinguish between translocation mechanisms that involve the direct penetration of the membrane core or pore formation from endocytic pathways involving the wrapping of nano-objects. For the design of a nano-object, it is crucial to consider that the translocation and endocytosis pathways lead to fundamentally different topological situations.

\section{A. Active transport}

Active transport refers to mechanisms that are enabled by an expenditure of chemical energy. Endocytotic pathways are widely associated with active processes ${ }^{25}$ since in biological environments dynamin catalyzes the separation step of an invagination. ${ }^{26,27}$ Grafting lipoproteins and other ligands onto nano-objects will make it possible to exploit active endocytic and phagocytic machineries of cells by binding to specific membrane receptors in the docking step.

Ion and glucose transporters are other common examples of protein channels that facilitate active translocation across a membrane. Proteins that can specifically transport synthetic nano-objects across a bilayer are missing. Developing such machinery will be particularly worthwhile because it has the potential to impart high selectivity onto translocation. Existing concepts related to passive polymer translocation through nanopore ${ }^{28}$ as well as voltage-driven DNA translocation through biological pores ${ }^{29}$ can be starting points for the development of translocation machineries for nano-objects driven by local chemical energy (adenosine triphosphate). It is thus crucial to more deeply study the mechanisms of existing transmembrane transporters and active lipid flip-flop catalyzing proteins. An interesting avenue of research could be aimed at identifying the minimal synthetic analogs or modifications of those proteins that enables them to bind to nano-objects and subsequently catalyze their translocation. Accurately predicting the catalytic role of active proteins interacting with nanostructures is an open field for molecular simulation techniques. Intervening in active and regulatory transport systems can, however, easily show the fate of over-ambition: A nanomaterial that perturbs active machineries such as glucose or ion transporters, ${ }^{30,31}$ or active lipid exchangers between leaflets, may cause unpredictable regulatory failures and toxic effects.

\section{B. Assisted transport}

Assisted translocation exploits global nonequilibrium processes or local response of the membrane that facilitate translocation processes, but that are not directly related to the translocation mechanism. A prototypical illustration of global nonequilibrium aspects is a translocation process that exploits the actively maintained lipid or protein asymmetry between the inner and outer monolayers. An interesting challenge is the possible transport of nano-objects driven by chemical potential differences-for instance, by developing analogs of secondary transporters. Other examples of assisted translocation include enhanced permeability at

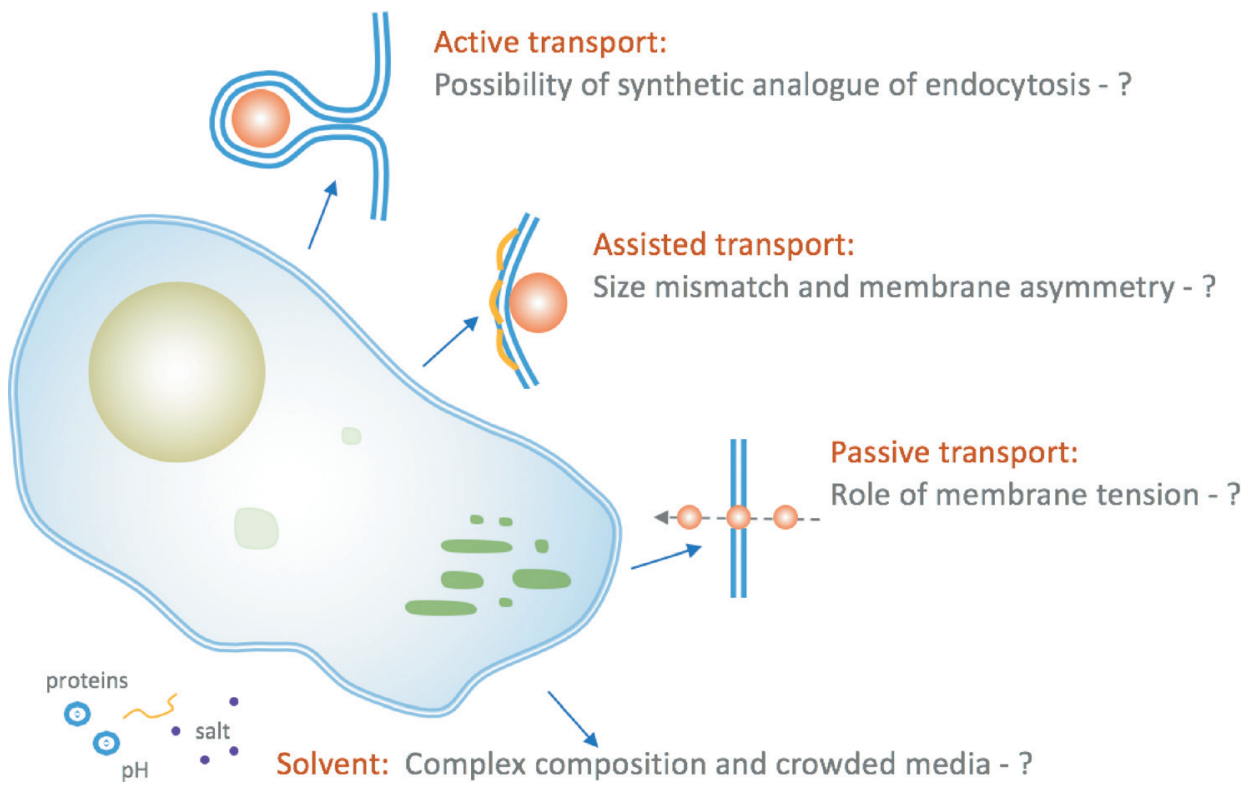

FIG. 1. Challenges and open questions in transport across a cell membrane. 
boundaries between lipid domains, ${ }^{32,33}$ the assistance by near-critical composition fluctuations or raftlike domains, ${ }^{34}$ as well as interfaces between lipids and membrane-inserted nano-objects with critical hydrophobicity. ${ }^{35}$ The adsorption of nano-objects at the membrane may locally alter the composition of the membrane in contact with the nano-object, and, in turn, facilitate translocation of the object. ${ }^{36}$

Endocytic pathways are assisted by families of curvature-inducing proteins that attach to the membrane: clathrin and bin, amphiphysin, rvs (BAR) proteins. Anisotropic and Janus nanoparticles can mimic curvatureinducing proteins, ${ }^{37,38}$ and promote the formation of invaginations. $^{39}$ In vitro experiments indicate that so-called $\mathrm{N}-\mathrm{BAR}$ proteins, by having a transmembrane domain, promote endocytosis in the absence of dynamin, while pure BAR-domains seem to restrict fission but support tubular shapes. ${ }^{40}$ On one hand, it is often discussed that specific assisting mechanisms are required for the final pinch-off to occur; on the other hand, computer simulations indicate that spontaneous endocytosis of wrapped nanoparticles also occurs in cases where N-BAR or equivalent molecules are not present. ${ }^{41-44}$ A key question here is how the barriers for altering membrane topology and concomitant time scales depend on the object enclosed. To this end, the prediction of the pinch-off dynamics and time scales can be seen as benchmark case for molecular simulation models. It is particularly challenging to map time scales and free energy barriers between atomistic and coarse-grained models-motivating the development of new simulation techniques bridging the gap. Beside computer simulation, it will be worthwhile to test existing theoretical models for the pinch-off ${ }^{45,46}$ and the role of "universal membrane remodelers" 47 via focused experiments with model membranes. An interesting question to address is the relation between nano-object size, and the spontaneous curvature induced by assisting proteins or synthetic analogs: can we predict matching sizes and shapes for selective transport?

An important aspect of nano-object transport attracting more attention is the role of cell membrane tension, which is on the order of $0.01 \mathrm{mN} / \mathrm{m}$ in native membranes. ${ }^{48}$ In many cases, the underlying actin cortex is also relevant by inducing a cortical tension on the order of $0.01-1 \mathrm{mN} / \mathrm{m} .{ }^{49}$ Experimental evidence shows that endocytic efficiency typically decreases with increasing membrane tension, but for some cell types the response can be inverted. ${ }^{50}$ Theoretically, it is expected that tension-induced restraining forces for particle wrapping appear for particle sizes larger than a characteristic length scale defined by the membrane bending rigidity and tension. ${ }^{51}$ For larger particles, the degree of wrapping is controlled by the competition between tension and adhesion. ${ }^{52}$ The release of membrane reservoirs $^{48}$ and membrane remodeling ${ }^{53}$ upon increasing tension or areas consumed by wrapping complicate the situation. Before disentangling all contributions in biological environments, however, it will be interesting to investigate wrapping and endocytosis as a function of tension in model experiments with reduced complexity. Membrane tension can also play a crucial role for translocation pathways across a membrane. The probability of transient pores induced, for instance, by cellpenetrating peptides is expected to be sensitive to the ratio between the tension of the membrane and the line tension of the pore. ${ }^{54}$

\section{Passive transport}

Passive translocation refers to the diffusion of small $(<10 \mathrm{~nm})$ nano-objects across the membrane, which is chiefly dictated by the properties of the nano-objects and their interactions with the membrane. Passive translocation processes do not require any external forces or gradients of other components between the two sides of the membrane. They are rather robust, fast, and present a universal platform for developing translocation approaches. The ratio between nanoobject size and the membrane thickness as well as its geometric shape are critical parameters. ${ }^{19,56}$ Additionally, the mechanical or chemical responsiveness of the nano-object, ${ }^{57}$ i.e., the deformability, and adaptability of the chemical surface composition and charge determine insertion and translocation. These surface properties of a nano-object can be controlled, e.g., by grafting polymers onto the surface of the nano-object. ${ }^{62}$ In particular, stimuli-responsive coatings with multicomponent brushes provide ample opportunities to tailor the passive translocation processes by environmental characteristics such as $\mathrm{pH}$, salt concentration, or temperature. ${ }^{55}$ Polymers in contact with a membrane may undergo conformational changes such as coil-globule transitions. ${ }^{58,59}$ It is suggested that some cell penetrating peptides switch to helical amphipathic structure in the presence of the membrane. ${ }^{60,61}$ To design synthetic analogs of these self-assembled peptides is a challenge.

In addition to passive translocation across the membrane, "passive endocytosis" was also hypothesized 63 and debated over several decades. ${ }^{64}$ The docking step and wrapping of nanoparticles has been described theoretically, ${ }^{51,65}$ and explored numerically as a function of shape and adhesion strength of the particle at the membrane. ${ }^{56,66}$ While an invagination can be driven by adhesion at the membrane as observed in model experiments, ${ }^{12}$ it remains an interesting question to what extent assisted or active processes are essential for the final pinch-off of the wrapped nano-object.

\section{CHALLENGES IN BIOLOGICALLY COMPLEX MEDIA}

Experimental and theoretical studies on the interactions between synthetic nanomaterials and membranes in biomimetic or in in vitro cellular systems often assume nano-objects of idealized shape, size, and surface in a simple fluid environment. Typical solvent environments considered are salt buffers such as phosphate-buffered saline (PBS) or water with a given concentration of monovalent ions. However, biological membranes are embedded in molecularly crowded aqueous environments, such as the cytoplasm and extracellular fluids, with distinct solvent conditions and compositional challenges that must be considered. 


\section{A. Properties of the surrounding media}

Ionic components, proteins, or RNA do not only determine simple physical properties such as $p \mathrm{H}$ and the screening of electrostatic interactions. Very recently, it has been discovered that several types of proteins together with RNA give rise to spatially controlled intracellular phase separation into droplets that are called RNA bodies or granules. ${ }^{67}$ Similarly, if foreign substances such as macromolecules, micelles, or nanoparticles are inserted into living systems, it is very likely that their properties and interactions with the cell membranes are different from those in simple aqueous solutions. For instance, bare nanoparticles can adsorb proteins and thus change their surface properties ${ }^{68}$ but polymers can also change their properties by adsorbing and binding components of the biological fluid. For instance, the puzzling phenomenon of passive translocation of positively charged arginine-rich peptides, and even of oligoarginines, was explained by the binding of (counter-)anions from the buffer. ${ }^{69}$ Few theoretical and simulation studies have taken into account complex formation between nano-objects and other components typical of biological solutions including counterion binding. The compensation of charge in polymers such as polypeptides can switch the monomer solubility from hydrophilic to hydrophobic since in many cases the uncharged backbone is hydrophobic. If nano-objects are close to the membrane, this binding process can be further influenced by the interaction with the membrane in particular by the charge and counterions located near the lipid head groups. It must be noted that arginine itself is positively charged and strongly hydrophilic which should prevent any passive pathway of these polymers through lipid bilayer membranes. Arginine-rich peptides such as trans-activator of transcription or homeoprotein transcription factors appear in nature and thus are evolutionarily optimized in the presence of biological fluids. Recently, it was demonstrated that cubeoctameric silsesquioxanes ${ }^{70}$ with similar positively charged ligands efficiently translocate through cell membranes.

Experimental evidence thus opens new possibilities for developing bioinspired cell-penetrating nano-objects, but also presents a challenge for theory and experiments using model membranes in artificial environments. A key question is: how many and which components of extracellular fluids (if considering the insertion process into the cell) are essential to mimic a typical extracellular environment in a representative way? Is there a standard for such a biological medium that is elementary enough to retain the advantages of minimal model systems? Is there a better standard for a biological medium than the typically used PBS suspensions $(p \mathrm{H} 7.4$ and physiological salt levels) to study nano-object membrane interactions? Nanomaterials represent length scales where the molecular crowding of cellular as well as intracellular environments substantially influence diffusion dynamics, excluded volume effects, and intermolecular association. ${ }^{71}$ A new standard medium should potentially contain crowding agents such as polyethylene glycol (PEG) or polysaccharides in order to simulate those effects. ${ }^{72}$
Another level of complexity arises when taking into account dynamically changing environments. As an example, during endocytic uptake of nanoparticles, the endosomal compartment is acidified, which can lead to the protonation of functional groups on a particle's surface, changing its net charge. In the endosome, this change in the $p \mathrm{H}$ of the environment is coupled with a change in the lipid composition of the interacting endosomal membrane, which can lead to significant changes in the nanomaterial's ability to disrupt or cross the membrane. ${ }^{73}$

Finally, recent attention has been paid to the dynamic feedback that membranes may induce in biological media via the recruitment of curvature-sensing proteins. It has been found that membrane curvature and cortical proteins can both take part in coupled oscillations of shape and concentration, ${ }^{74,75}$ which presumably contribute to cell signaling processes. How would a nanomaterial interfere with those dynamics?

\section{B. Protein corona}

In physiological environments, a large number of proteins and other biomolecules are present. These molecules can rapidly bind in a temporally complex way to nano-objects, and form fluctuating coronas around nano-objects that may have a strong influence on their interactions with a biological environment. ${ }^{76-79}$ In analogy with the Vroman effect, ${ }^{80,81}$ the composition of coronas may vary dramatically over time. ${ }^{82}$

Nanoparticles that are immersed in human blood serum have coronas that consist of proteins such as albumin, immunoglobulins, fibrinogen, apolipoproteins, and proteins from the complement system. ${ }^{9,83,84}$ There is a large class of proteins called opsonins that label foreign objects to be detected by the immune system, and trigger the uptake by phagocytes and macrophages. In contrast, another class of proteins, dysopsonins, including albumins and apolipoproteins are known to inhibit phagocytic uptake. ${ }^{76,85}$ The composition of both groups adsorbed at nanocarriers in blood serum controls their elimination by resident macrophages. ${ }^{86,87}$ Recent experiments, for instance, seem to explain the so-called stealth effect of PEG coatings against phagocytosis by the selective adsorption of lipoproteins and apolipoproteins onto the PEGcoated nanocarriers. ${ }^{88}$ However, the hypothesis that PEGylation of particles increases the binding of dysopsonins that mask the particles was already put forward more than 15 years ago. ${ }^{89}$ Since corona formation seems almost unavoidable, the central challenge is to control its composition and structure as a function of time.

\section{Real biomembranes}

Lipid bilayers can be convenient model systems for nanoparticle-membrane interactions that enable detailed physical insight to be obtained thanks to their relative structural simplicity and well-characterized properties. However, real biological membranes are far more complex in structure, containing a large amount of both integral and peripheral 
proteins $^{90,91}$ in addition to a high degree of glycosylation providing a complex coating with polymeric sugars. Further complexity is added by the cell membrane's transmembrane asymmetry, lateral heterogeneities, and underlying cytoskeleton, which is a dynamic network of semiflexible to rigid polymers. Future theoretical and experimental model systems should start to take this increased membrane complexity into account in order to understand the true extent to which a lipid bilayer can model nanoparticle interactions at a real biomembrane. For example, giant unilamellar vesicles can be fabricated directly from the plasma membranes of mammalian cells and are known as giant plasma membrane vesicles (GPMVs). ${ }^{92}$ They contain most of the natural components of a real cell membrane but without the active processes of a real cell. Therefore, these materials are ideal experimental systems to bridge the gap between model lipid membranes and the whole cell. Experiments with GPMVs not only allow the validity of more abstract theoretical and experimental models to be tested, but can be a starting point to study the effects of protein and lipid sorting as well as more specific coupling of nanomaterials with biomolecular interaction networks. Although structurally impaired compared to GPMVs, planar supported membranes made from native cell membranes serve as additional model systems, which allow for a large arsenal of sophisticated surface analytical tools. ${ }^{93,94}$

A further challenge arises in the design of nanoparticles that target a specific cell type. This is particularly important for nanomedicine applications, where drug-loaded particles might be targeted to a specific subpopulation of cells possessing a particular disease pathology. In many disease states, e.g., cancers, it is known that cells upregulate specific cell surface receptors such that they are then present in higher concentrations within the plasma membrane. ${ }^{95}$ Among many others, well-known examples include growth factor receptors, ${ }^{96}$ vitamin receptors such as folate receptors ${ }^{97}$ and the transferrin receptor. ${ }^{98}$ Receptor overexpression is usually heterogeneous within different cells of a single tumor and also between different patients for a given type of cancer, posing a fundamental challenge when aiming for generalized descriptions of molecular and physical mechanisms of how nano-objects engage in receptor binding. Targeting approaches have involved the attachment of high affinity ligands to the surface of a nanoparticle that targets these receptors. However, receptors that are overexpressed in disease state are also present in the membranes of healthy cells, albeit at lower concentrations, leaving significant chances for off-target binding to healthy cells. Therefore, we see a central challenge to clarify the effect of ligand density on nano-objects on receptor-mediated uptake. Complementary to this challenge, the surface density of receptors needs attention as playing a role for nanoparticle targeting to diseased cells. An additional question for in vitro systems that are barely addressed in current mechanistic studies, but likely important to unravel the uptake process of nanoparticles, is the impact of hydrodynamic interactions in biological fluid flows on cell-specific adhesion.

\section{CONCLUSIONS}

In this Perspective, we illustrate the progress and collect open questions related to the design and function of nanomaterials that interact with lipid and biological membranes (Table I). The minimalistic but well-controlled design approach used by scientists is conceptually different from biological adaptation and evolution. The mismatch between theoretical or experimental models of reduced complexity and the multitude of interactions concerted within rich biological environments makes it challenging to design functional materials. There has been substantial progress in all fields related to nanomaterial transport across membranes, including the development of theoretical and experimental models, nanomaterial synthesis, and in vitro testing in systems with increased biological complexity. Both endocytic pathways as well as translocation by penetrating through a bilayer have been extensively analyzed via theoretical

TABLE I. Summary of challenges and open questions.

Challenges Next levels of model complexity.

Missing links to biological context

How do chemical properties translate into

physical control parameters within complex media?

Statistical nature of nano-object properties such as polydispersity, in-sample variations of surface shape and composition.

Challenges for transport across lipid bilayer membranes

How to exploit protein complexes

for specific nano-object transport?

Can we rationalize dynamic barriers for topological

membrane transitions as a function of molecular

composition and curvature-inducing nano-objects?

Include active components in molecular models.

Systematically study the role of membrane tension in simulation studies and model experiments.

Challenges in biologically complex media

How to mimic cellular and intracellular fluids

in a representative way? Is there a better standard

for a model biological media than PBS?

Can we control protein coronas as a function of time?

Fluid complexity includes granules, specific counter-ion

condensation, cononsolvency, dynamics in composition and $p \mathrm{H}$, effects of molecular crowding.

Binding and interaction of membranes with cytoskeleton. 
models, computer simulation, and experimental studies. On the other side, chemists are able to synthesize highly advanced materials with dynamic control of attached ligands ("molecular surgery"8) and can monitor the transport of complex materials through biological membranes. ${ }^{76}$ Even the molecular details of the immune response induced by polymer-based coatings and proteins have recently been more clearly defined. ${ }^{88}$ For further progress in the design flow between theory and in vitro testing, we emphasize the potential to close missing links between model systems and the biological context. Theoretical and experimental model systems may systematically include next levels of complexity: active components such as enzymes, solvent complexity and cosolvency, the nano-object's interplay with proteins by means of protein corona and curvature-inducing proteins, the variation of membrane tension, coupling to the cytoskeleton, and the lateral structure of multicomponent membranes. Systematically designed model experiments may receive more emphasize before the in vitro testing of newly synthesized materials. For example, existing theoretical models for adhesion- and tension-dependent wrapping have not been extensively tested yet in model experiments. An interesting phenomenon to understand on a physical molecular level will be the membrane fission event during endocytosis. We illustrate the importance of integrating the existing knowledge on membrane fusion, vesicle formation by membrane fission, and vesicle transport into a complete picture of the whole endocytic/exocytic cycle. Precisely determining the topological pathway of a nano-object is crucial for knowing which sensitive parts of a cell, e.g., DNA, are exposed to the object for causing potentially toxic effects. It is important not to over-define the targeted functionality, and to avoid aiming for multifunctionality. Instead, it would be advantageous to require the nanomaterial to be as minimally specific as necessary in order to act as delivery vector, nanosensor, or imaging agent. Finally, we close by throwing two challenging questions: Can we create a synthetic analog of a complete endocytic cycle? Can we adapt a synthetic analog of active ion transporters for direct translocation of nanoobjects?

\section{ACKNOWLEDGMENTS}

This discussion is inspired by the 5th Workshop on Biomaterials and their Interactions with Biological and Model Membranes 2016 held in Salou http:// meeting.softmat.net/. M.W. acknowledge funding from Marie Curie Actions under European Union 7th Framework Programme (FP7) Initial Training Network SNAL 608184. E.P.P. acknowledges the financial support by the DFG within the SFB 1032 TP B01 and A09. M.M. acknowledges financial support by the DFG within the SFB 937 TP A07. J.B.F. acknowledges financial support by the DFG within the SFB 1027 TP B4. H.K. acknowledges financial support by the DFG (Nos. KR 3524/4-1 and INST 91/289-1 FUGG). F.H. acknowledges the Swedish Foundation for Strategic Research (SSF) Grant No. IRC15-0065. P.A.B. acknowledges funding from the E.U. H2020 HISENTS project (GA No. 685817) and EPSRC Grant No. EP/ M027929/1. The authors acknowledge fruitful discussions and critical reading of the manuscript by Eric R. Dufresne as well as inspiring discussions with Elena P. Ivanova.

${ }^{1}$ P. A. Dhawan, V. Sharma, and D. Parmar, Nanotoxicology 3, 1 (2009).

${ }^{2}$ L. Stander and L. Theodore, Int. J. Environ. Res. Public Health 8, 470 (2011)

${ }^{3}$ A. E. Nel, L. Mädler, D. Velegol, T. Xia, E. M. V. Hoek, P. Somasundaran, F. Klaessig, V. Castranova, and M. Thompson, Nat. Mater. 8, 543 (2009)

${ }^{4}$ S. Mitragotri and J. Lahann, Nat. Mater. 8, 15 (2009).

${ }^{5}$ S. Behzadi et al., Chem. Soc. Rev. 46, 4218 (2017).

${ }^{6}$ J. Deng and C. Gao, Nanotechnology 27, 412002 (2016).

${ }^{7}$ D. Vollath, Nanomaterials: An Introduction to Synthesis, Properties and Applications, 2nd ed. (Wiley-VCH, Weinheim, Germany, 2013).

${ }^{8}$ Q. Li, T.-Y. Luo, M. G. Taylor, S. Wang, X. Zhu, Y. Song, G. Mpourmpakis, N. L. Rosi, and R. Jin, Sci. Adv. 3, e1603193 (2017).

${ }^{9}$ T. Cedervall, I. Lynch, S. Lindman, T. Berggård, E. Thulin, H. Nilsson, K. A. Dawson, and S. Linse, Proc. Natl. Acad. Sci. 104, 2050 (2007).

${ }^{10}$ H. Zhang et al., ACS Nano 6, 4349 (2012).

${ }^{11}$ B. Fahmy and S. A. Cormier, Toxicol. In Vitro 23, 1365 (2009).

${ }^{12}$ Y. Yu and S. Granick, J. Am. Chem. Soc. 131, 14158 (2009).

${ }^{13}$ S. Zhang, A. Nelson, and P. A. Beales, Langmuir 28, 12831 (2012).

${ }^{14}$ N. B. Leite, A. Aufderhorst-Roberts, M. S. Palma, S. D. Connell, J. Ruggiero Neto, and P. A. Beales, Biophys. J. 109, 936 (2015).

${ }^{15}$ B. Wang, L. Zhang, S. C. Bae, and S. Granick, Proc. Natl. Acad. Sci. 105, 18171 (2008).

${ }^{16}$ S. W. Hell and J. Wichmann, Opt. Lett. 19, 780 (1994).

${ }^{17}$ V. Westphal, S. O. Rizzoli, M. A. Lauterbach, D. Kamin, R. Jahn, and S. W. Hell, Science 320, 246 (2008).

${ }^{18}$ A. Brown and N. Hondow, Front. Nanosci. 5, 95 (2013).

${ }^{19}$ Y. Guo, E. Terazzi, R. Seemann, J. B. Fleury, and V. A. Baulin, Sci. Adv. 2, e1600261 (2016).

${ }^{20}$ C. M. Beddoes, C. P. Case, and W. H. Briscoe, Adv. Colloid Interface Sci. 218, 48 (2015)

${ }^{21}$ H. Ding and Y. Ma, Small 11, 1055 (2015).

${ }^{22}$ A. Verma and F. Stellacci, Small 6, 12 (2010).

${ }^{23}$ S. Pogodin, M. Werner, J.-U. Sommer, and V. A. Baulin, ACS Nano 6, 10555 (2012).

${ }^{24}$ M. Fuhrmans, G. Marelli, Y. G. Smirnova, and M. Müller, Chem. Phys. Lipids 185, 109 (2015).

${ }^{25}$ S. L. Schmid, J. Cell Biol. 111, 2307 (1990).

${ }^{26}$ R. Ramachandran, Semin. Cell Dev. Biol. 22, 10 (2011)

${ }^{27}$ W.-D. Zhao, E. Hamid, W. Shin, P. J. Wen, E. S. Krystofiak, S. A. Villarreal, H.-C. Chiang, B. Kachar, and L.-G. Wu, Nature 534, 548 (2016).

${ }^{28}$ W. Sung and P. J. Park, Phys. Rev. Lett. 77, 783 (1996).

${ }^{29}$ G. F. Schneider and C. Dekker, Nat. Biotechnol. 30, 326 (2012).

${ }^{30}$ Z. Yang, Z. W. Liu, R. P. Allaker, P. Reip, J. Oxford, Z. Ahmad, and G. Ren, J. R. Soc. Interface 7, S411 (2010).

${ }^{31}$ S. L. Bryant, J. E. Eixenberger, S. Rossland, H. Apsley, C. Hoffmann, N. Shrestha, M. McHugh, A. Punnoose, and D. Fologea, J. Nanobiotechnol. 15, 90 (2017).

${ }^{32}$ L. Yang and J. T. Kindt, J. Phys. Chem. B 120, 11740 (2016).

${ }^{33}$ A. Tiwari, A. Prince, M. Arakha, S. Jha, and M. Saleem, Nanoscale 10, 3369 (2018)

${ }^{34}$ K. Wodzinska, A. Blicher, and T. Heimburg, Soft Matter 5, 3319 (2009).

${ }^{35}$ H. Rabbel, M. Werner, and J.-U. Sommer, Macromolecules 48, 4724 (2015).

${ }^{36}$ C. L. Bergstrom, P. A. Beales, Y. Lv, T. K. Vanderlick, and J. T. Groves, Proc. Natl. Acad. Sci. 110, 6269 (2013).

${ }^{37}$ Y. Schweitzer, T. Shemesh, and M. M. Kozlov, Biophys. J. 109, 564 (2015).

${ }^{38}$ P. A. Beales, B. Ciani, and A. J. Cleasby, Phys. Chem. Chem. Phys. 17, 15489 (2015).

${ }^{39}$ J. Agudo-Canalejo and R. Lipowsky, Nano Lett. 15, 7168 (2015).

${ }^{40}$ E. Boucrot, A. Pick, G. Çamdere, N. Liska, E. Evergren, H. T. McMahon, and M. M. Kozlov, Cell 149, 124 (2012). 
${ }^{41}$ H. Yuan, C. Huang, J. Li, G. Lykotrafitis, and S. Zhang, Phys. Rev. E 82, 011905 (2010).

${ }^{42}$ K. Yang and Y. Ma, Soft Matter 8, 606 (2012).

${ }^{43}$ R. Vácha, F. J. Martinez-Veracoechea, and D. Frenkel, Nano Lett. 11, 5391 (2011).

${ }^{44}$ C. Huang, Y. Zhang, H. Yuan, H. Gao, and S. Zhang, Nano Lett. 13, 4546 (2013).

${ }^{45}$ Y. Kozlovsky and M. M. Kozlov, Biophys. J. 85, 85 (2003).

${ }^{46}$ G. Zhang and M. Müller, J. Chem. Phys. 147, 064906 (2017).

${ }^{47}$ M. M. Kozlov, H. T. McMahon, and L. V. Chernomordik, Trends Biochem. Sci. 35, 699 (2010).

${ }^{48}$ J. Dai, M. P. Sheetz, X. Wan, and C. E. Morris, J. Neurosci. 18, 6681 (1998).

${ }^{49}$ M. Herant, V. Heinrich, and M. Dembo, J. Cell Sci. 118, 1789 (2005).

${ }^{50}$ G. Apodaca, Am. J. Physiol. Renal Physiol. 282, F179 (2002).

${ }^{51}$ M. Deserno and T. Bickel, Europhys. Lett. 62, 767 (2003).

${ }^{52}$ M. Deserno and W. M. Gelbart, J. Phys. Chem. B 106, 5543 (2002).

${ }^{53}$ A. J. Kosmalska et al., Nat. Commun. 6, 7292 (2015).

${ }^{54}$ C. Taupin, M. Dvolaitzky, and C. Sauterey, Biochemistry 14, 4771 (1975).

${ }^{55}$ F. Léonforte and M. Müller, ACS Appl. Mater. Interfaces 7, 12450 (2015).

${ }^{56}$ S. Dasgupta, T. Auth, and G. Gompper, Nano Lett. 14, 687 (2014).

${ }^{57}$ R. C. Van Lehn and A. Alexander-Katz, Soft Matter 7, 11392 (2011).

${ }^{58}$ C. Herold, P. Schwille, and E. P. Petrov, Phys. Rev. Lett. 104, 148102 (2010).

${ }^{59}$ A. G. Cherstvy and E. P. Petrov, Phys. Chem. Chem. Phys. 16, 2020 (2014).

${ }^{60}$ S. Deshayes, T. Plénat, G. Aldrian-Herrada, G. Divita, C. Le Grimellec, and F. Heitz, Biochemistry 43, 7698 (2004).

${ }^{61}$ W. B. Kauffman, T. Fuselier, J. He, and W. C. Wimley, Trends Biochem. Sci. 40, 749 (2015).

${ }^{62}$ H.-M. Ding and Y.-Q. Ma, Sci. Rep. 6, 26783 (2016).

${ }^{63}$ D. W. Fawcett and D. A. Stagg, J. Submicrosc. Cytol. 18, 11 (1986).

${ }^{64}$ M. K. Shaw, L. G. Tilney, and A. J. Musoke, J. Cell Biol. 113, 87 (1991).

${ }^{65}$ H. Gao, W. Shi, and L. B. Freund, Proc. Natl. Acad. Sci. U. S. A. 102, 9469 (2005).

${ }^{66}$ A. H. Bahrami, M. Raatz, J. Agudo-Canalejo, R. Michel, E. M. Curtis, C. K. Hall, M. Gradzielski, R. Lipowsky, and T. R. Weikl, Adv. Colloid Interface Sci. 208, 214 (2014).

${ }^{67}$ C. P. Brangwynne, P. Tompa, and R. V. Pappu, Nat. Phys. 11, 899 (2015).

${ }^{68}$ M. Rahman, S. Laurent, N. Tawil, L. Yahia, and M. Mahmoudi, ProteinNanoparticle Interaction (Springer, Berlin/Heidelberg, 2013), pp. 21-44.

${ }^{69}$ N. Sakai, S. Futaki, and S. Matile, Soft Matter 2, 636 (2006).

${ }^{70}$ S. Hörner, S. Knauer, C. Uth, M. Jöst, V. Schmidts, H. Frauendorf, C. M. Thiele, O. Avrutina, and H. Kolmar, Angew. Chem. Int. Ed. 55, 14842 (2016).

${ }^{71}$ R. J. Ellis, Trends Biochem. Sci. 26, 597 (2001).
${ }^{72}$ N. Tokuriki, M. Kinjo, S. Negi, M. Hoshino, Y. Goto, I. Urabe, and T. Yomo, Protein Sci. 13, 125 (2004).

${ }^{73}$ S. C. Goodchild, T. Sheynis, R. Thompson, K. W. Tipping, W.-F. Xue, N. A. Ranson, P. A. Beales, E. W. Hewitt, and S. E. Radford, PLoS One 9, e104492 (2014).

${ }^{74}$ M. Wu, X. Wu, and P. D. Camilli, Proc. Natl. Acad. Sci. 110, 1339 (2013).

${ }^{75}$ Z. Wu, M. Su, C. Tong, M. Wu, and J. Liu, Nat. Commun. 9, 136 (2018).

${ }^{76}$ D. Docter, D. Westmeier, M. Markiewicz, S. Stolte, S. K. Knauer, and R. H. Stauber, Chem. Soc. Rev. 44, 6094 (2015).

${ }^{77}$ S. R. Saptarshi, A. Duschl, and A. L. Lopata, J. Nanobiotechnol. 11, 26 (2013).

${ }^{78}$ M. P. Monopoli, C. Åberg, A. Salvati, and K. A. Dawson, Nat. Nanotechnol. 7, 779 (2012).

${ }^{79}$ C. D. Walkey, J. B. Olsen, F. Song, R. Liu, H. Guo, D. W. H. Olsen, Y. Cohen, A. Emili, and W. C. W. Chan, ACS Nano 8, 2439 (2014).

${ }^{80}$ L. Vroman, Bull. N. Y. Acad. Med. 64, 352 (1988).

${ }^{81}$ S. M. Slack and T. A. Horbett, Proteins Interfaces II, edited by T. A. Horbett and J. L. Brash (American Chemical Society, Washington, DC, 1995), pp. 112-128.

${ }^{82}$ E. Casals, T. Pfaller, A. Duschl, G. J. Oostingh, and V. Puntes, ACS Nano 4, 3623 (2010).

${ }^{83}$ C. D. Walkey and W. C. W. Chan, Chem. Soc. Rev. 41, 2780 (2012).

${ }^{84}$ M. Lundqvist, J. Stigler, G. Elia, I. Lynch, T. Cedervall, and K. A. Dawson, Proc. Natl. Acad. Sci. 105, 14265 (2008).

${ }^{85}$ D. R. Absolom, Methods Enzymol. 132, 281 (1986).

${ }^{86}$ T. Ishida, H. Harashima, and H. Kiwada, Biosci. Rep. 22, 197 (2002).

${ }^{87}$ D. Lombardo, P. Calandra, D. Barreca, S. Magazù, and M. Kiselev, Nanomaterials 6, 125 (2016).

${ }^{88}$ S. Schöttler, G. Becker, S. Winzen, T. Steinbach, K. Mohr, K. Landfester, V. Mailänder, and F. R. Wurm, Nat. Nanotechnol. 11, 372 (2016).

${ }^{89}$ M. Vert and D. Domurado, J. Biomater. Sci. Polym. Ed. 11, 1307 (2000).

${ }^{90}$ A. D. Dupuy and D. M. Engelman, Proc. Natl. Acad. Sci. U. S. A. 105, 2848 (2008).

${ }^{91}$ D. M. Engelman, Nature 438, 578 (2005).

${ }^{92}$ E. Sezgin, H.-J. Kaiser, T. Baumgart, P. Schwille, K. Simons, and I. Levental, Nat. Protoc. 7, 1042 (2012).

${ }^{93}$ H. Pace, L. Simonsson Nyström, A. Gunnarsson, E. Eck, C. Monson, S. Geschwindner, A. Snijder, and F. Höök, Anal. Chem. 87, 9194 (2015).

${ }^{94}$ M. J. Richards, C.-Y. Hsia, R. R. Singh, H. Haider, J. Kumpf, T. Kawate, and S. Daniel, Langmuir 32, 2963 (2016).

${ }^{95}$ M. J. Akhtar, M. Ahamed, H. A. Alhadlaq, S. A. Alrokayan, and S. Kumar, Clin. Chim. Acta 436, 78 (2014).

${ }^{96}$ A. M. Master and A. Sen Gupta, Nanomedicine 7, 1895 (2012).

${ }^{97}$ C. Marchetti et al., OncoTargets Ther. 7, 1223 (2014).

${ }^{98}$ T. R. Daniels et al., Biochim. Biophys. Acta 1820, 291 (2012). 